\title{
La CIE debería de eliminar la especificación etiológica en la mayoría de diagnósticos atribuibles al alcohol
}

\section{For most fully alcohol-attributable diagnoses in the ICD, the etiological specification should be removed}

\author{
Shannon LangE*,**, Michael Roerecke*,***,****, Jürgen ReHM*, **, ***, ****, *****, $* * * * * *$, \\ $* * * * * * *$
}

\begin{abstract}
* Instituto de Investigación sobre las políticas de salud mental, Centro de Adicciones y Salud Mental, 33 Russell St., Toronto, ON Canadá, M5S 2S1

** Instituto de Ciencias Médicas, Universidad de Toronto, 1 King's College Cir., Toronto, Ontario, Canadá, M5S 1A8

*** Escuela de Salud Pública Dalla Lana, Universidad de Toronto, 155 College St., Toronto, Ontario, Canadá, M5T 3M7

**** Instituto Familia Campbell de Investigación sobre la Salud Mental, Centro de Adicciones y Salud Mental, 33 Russell Street, Toronto, Ontario, Canadá, M5S 2S1

***** Departamento de Psiquiatría, Universidad de Toronto, 250 College Street, Toronto, Ontario, Canadá M5T 1R8

****** Instituto de Psicología Clínica y Psicoterapia y Centro de Epidemiología Clínica y Estudios Longitudinales, Technische Universität Dresden, Chemnitzer Str. 46, D-01187 Dresden, Alemania

******* Departamento de Proyectos de Salud Internacionales, Instituto de Liderazgo y Gestión de la Salud, I.M. Sechenov First Moscow State Medical University, Leontyevsky Pereulok 9, 125009 Moscú, Federación de Rusia
\end{abstract}

L a Clasificación Internacional de Enfermedades (CIE) es el estándar internacional para definir y reportar enfermedades y condiciones de salud, con el fin de servir como base para identificar estadísticas sanitarias y tendencias a nivel mundial además de tomar decisiones basadas en la evidencia (Organización Mundial de la Salud, s.a.). Hay más de 40 enfermedades $100 \%$ atribuibles al alcohol (Rehm et al., 2017) en las versiones actuales de la CIE (10. ${ }^{\mathrm{a}}$ revisión [CIE-10] y 11. a revisión [CIE-11]) (Organización Mundial de la Salud, 2018a). La costumbre de especificar «alcohol», «alcohólico» o «inducido por el alcohol» en el nombre de una enfermedad en la CIE se viene haciendo desde la década de los veinte del siglo pasado. No obstante, ha llegado el momento para reconsiderar esta práctica para la mayoría de las enfermedades y las categorías de lesiones, con la excepción de los trastornos por uso del alcohol (CIE-11: 6C40) o la intoxicación por alcohol (CIE-11: NE61, PD00, PH50), dado que la práctica no suele resultar en una intervención clínica específica, sino que implica un número de consecuencias negativas. Nuestra argumentación defiende que la especifi- cación de la etiología debe eliminarse de la mayoría de los nombres de los diagnósticos completamente atribuibles al alcohol en la CIE, y presentamos dos ejemplos concretos para mostrar las consecuencias de esta práctica: hepatopatía alcohólica (CIE-11: DB94) y síndrome del espectro alcohólico fetal (SAF; ICD-11: LD2F.00).

\section{Hepatopatía alcohólica}

Primero, los diagnósticos de todas las categorías con «alcohol» en su nombre son notablemente subestimados, tanto en el sistema de atención sanitaria como en los certificados de defunción. Veamos como ejemplo el estudio clásico de Puffer y Griffith (1967), que incluyó datos de 12 ciudades en diez países, y comparó los datos de los certificados de defunción con los datos de los registros hospitalarios y las entrevistas con los médicos tratantes y los familiares. Esto resultó en multiplicar por más de dos el número de muertes consideradas debidas a la cirrosis hepática alcohólica, donde la mayoría de estos casos nuevos se registraron originalmente bajo otras categorías de cirrosis, ninguna de

Recibido: Julio 2019; Aceptado: Noviembre 2019.

Enviar correspondencia a: Shannon Lange, $\mathrm{PhD}$

Institute for Mental Health Policy Research. Centre for Addiction and Mental Health. 33 Russell St., Room T521, Toronto, ON Canada M5S 2S1. Tel: +1 4165358501 ext. 34512. Email: shannon.lange@camh.ca 
las cuales apuntó al alcohol como agente causal. Este tipo de infradeclaración continúa en la actualidad y no se restringe a la hepatopatía alcohólica, sino que abarca otras enfermedades crónicas completamente atribuibles al alcohol (véase ejemplos en Rehm, Hasan, Imtiaz y Neufeld, 2017). Esto se ha demostrado usando varios estándares de comparación, autopsias, marcadores clínicos y entrevistas con los familiares, además de medidas indirectas. Uno de los motivos principales detrás de esta infradeclaración es que los diagnósticos con alcohol se asocian con una mayor estigmatización, más allá del estigma de los trastornos mentales (Schomerus et al., 2011). Existe la idea que las personas que beben alcohol en exceso y que tienen trastornos de uso de alcohol son, además de responsables de su trastorno, agresivos y disruptivos. Este estigma puede provocar que los bebedores en exceso eviten el sistema de atención sanitaria y, en última instancia, no revelen su consumo de alcohol (Probst, Manthey, Martinez y Rehm, 2015).

La mayoría de los tratamientos para las enfermedades hepáticas son iguales, independientemente de su etiología: en otras palabras, no necesariamente hay diferencias en las intervenciones para el hígado, y el uso de alcohol siempre debería ser valorado, minimizado o evitado. Los biomarcadores modernos, como los fosfatidiletanoles (PEth) (Carvalho, Heilig, Perez, Probst y Rehm, 2019; Andresen-Streichert, Müller, Glahn, Skopp y Sterneck, 2018), pueden usarse para valorar el uso de alcohol y así evitar la posible infradeclaración como resultado de la estigmatización. El motivo por el cual se ha de intervenir cuando se reporta el uso de alcohol es que el consumo regular de incluso pequeñas cantidades de alcohol puede provocar la muerte en las personas afectadas por la cirrosis hepática alcohólica (Fuster y Samet, 2018). Desafortunadamente, el uso de alcohol suele quedar sin abordar cuando se ignora la etiología de una enfermedad. Esto es problemático, dado que un estudio reciente de todos los pacientes franceses durante un periodo de cinco años mostró que el 71,8\% (IC 95\%: 66,0\% a 76.8\%) de 17669 complicaciones relacionadas con el hígado, el 67,4\% (IC 95\%: 61,6\% a 72,4\%) de 1599 trasplantes de hígado, y el 68,8\% (IC 95\%: 63,4\% a $73,5 \%$ ) de 6677 muertes de personas con infecciones crónicas del virus de hepatitis $\mathrm{C}$ eran atribuibles al uso de alcohol, y que una gran parte podían haberse evitado si se hubiese abordado el uso de alcohol (Schwarzinger, Baillot, Yazdanpanah, Rehm y Mallet, 2017). Las cifras anteriores incluso pueden estar subestimadas por los motivos mencionados anteriormente: el uso del alcohol no se valora ni reporta de manera regular en los hospitales en Francia, y es probable que se hayan infradeclarado en nuestro estudio los trastornos usados para identificar un consumo excesivo de alcohol. Este razonamiento también aplica a las llamadas enfermedades del hígado graso no alcohólico (ICD-11: DB92), en las cuales el uso de alcohol también asume un papel en su empeoramiento (Fuster y Samet, 2018).
Por último, la dependencia de la hepatopatía alcohólica como categoría supone un impedimento severo a la estimación del impacto real del alcohol porque las personas se clasifican en base a la presunción de una etiología y no por el impacto del alcohol como factor de riesgo. Por ejemplo, un análisis que dependió del diagnóstico de cirrosis hepática alcohólica para estimar la proporción de mortalidad por cirrosis hepática y la carga de la enfermedad atribuible al alcohol (GBD 2016 Alcohol Collaborators, 2018) obtuvo unas estimaciones de mortalidad y carga de la enfermedad un $50 \%$ y $60 \%$ más bajos, respectivamente, que otro análisis que se basó en todos las cirrosis hepáticas y que calculó la contribución del alcohol mediante la metodología de la fracción atribuible al alcohol habitual en el Informe sobre la situación mundial del alcohol y la salud (Organización Mundial de la Salud, 2018b). Es más, la distinción entre enfermedad hepática alcohólica o no alcohólica suele basarse en el consumo de alcohólica informado por el paciente. El umbral varía entre 20-40 gramos de etanol puro por día. No obstante, aparte de que la mayoría de las personas son incapaces de informar fiablemente sobre su consumo de alcohol, o simplemente mienten sobre ello, este umbral parece ser arbitrario y no tiene en cuenta la naturaleza multifactorial de la etiología de las enfermedades hepáticas (Pimpin et al., 2018; Roerecke et al., 2019). De nuevo, el uso de los biomarcadores, como el PEth, sería recomendable tanto para la práctica clínica como para la investigación.

Esto no significa que el alcohol no sea uno de los factores de riesgo principales de las enfermedades hepáticas, pero identificar una enfermedad hepática «alcohólica» ignora la contribución de otros factores de riesgo y, a la inversa, la contribución del alcohol es ignorado en las llamadas enfermedades hepáticas «no alcohólicas».

\section{Síndrome del espectro alcohólico fetal (SAF)}

Como ocurre en los diagnósticos completamente atribuibles al alcohol, las personas que han tenido una exposición prenatal al alcohol a menudo piensan que otros les juzgan y, porque evitan ser etiquetados con un diagnóstico que les estigmatiza, no acuden a servicios diagnósticos e intervenciones que podrían contribuir a mejorar su calidad de vida. El estigma es un factor de riesgo clínico importante, dado que se sabe que atrase la búsqueda de tratamiento, empeora su curso y evolución, reduce el cumplimiento y aumenta el riesgo de recaída, lo que provoca aún más discapacidad, discriminación y aislamiento, incluso en las personas que han tenido acudido a los servicios (Shrivastava, Bureau, Rewari y Johnston, 2013). Es por esta razón que las mujeres tienden a negar o infradeclarar su uso de alcohol durante el embarazo (Lange, Shield, Koren, Rehm y Popova, 2014), lo que, en última instancia, resulta en errores diagnósticos respecto del FAS. El propósito del sistema de 
clasificación es ofrecer categorías de trastornos independientes (Lecrubier, 2008); no obstante, la coexistencia del FAS con diagnósticos de otros trastornos del neurodesarrollo (por ejemplo, el trastorno por déficit de atención con hiperactividad [CIE-11: 6A05]) parece ser la norma (Lange, Rehm, Anagnostou y Popova, 2018). De hecho, se encontró hace poco que los niños con FAS (un término general que engloba a varios diagnósticos relacionados con el alcohol, incluyendo el FAS) no pueden diferenciarse de otros niños con otros trastornos del neurodesarrollo en términos de su neurodesarrollo y conducta (Lange, Shield, Rehm, Anagnostou y Popova, 2019). Este hallazgo es un ejemplo del sinsentido de especificar «alcohol» como la causa de los trastornos del neurodesarrollo en la práctica clínica, especialmente porque no hay evidencia que sustente dicha diferenciación con respecto a la efectividad del tratamiento (Premji, Benzies, Serrett y Hayden, 2007). La exposición prenatal al alcohol se asocia con un amplio rango de síntomas y, como tal, un diagnóstico de FAS no ofrece una serie de síntomas específicos del individuo e, incluso peor, no contribuye hacia un plan de tratamiento definido (Price y Miskelly, 2015), dada la ausencia de una estrategia terapéutica concreta para el FAS (Murawski, Moore, Thomas y Riley, 2015).

\section{Discusión}

Los dos ejemplos anteriores dejan claro que especificar «alcohol» en el nombre de una enfermedad tiene una relevancia clínica limitada, e incluso puede atrasar la atención médica y llevar a un tratamiento erróneo. Dicha especificación puede resultar en informes inadecuados, lo que tiene implicaciones significativas para la investigación, las políticas de salud pública y la planificación de la atención sanitaria, en particular porque, según indica toda la evidencia, se infradeclararían las condiciones que contienen «alcohol» en su nombre.

Ciertamente, puede argumentarse que especificar «alcohol» en el nombre de una enfermedad es necesario para maximizar los esfuerzos preventivos. De hecho, las tasas de incidencia y prevalencia de una condición son indicadores de la carga de las respectivas enfermedades en la salud pública, y sirven como base para la asignación de recursos a la atención sanitaria y las iniciativas preventivas. No obstante, si dichas estimaciones son erróneas porque se basan en un sistema que intrínsecamente lleva a un diagnóstico erróneo, entonces se debe deducir que el sistema mismo es defectuoso. Dado que la CIE no tiene como objetivo maximizar los esfuerzos de prevención porque es un estándar internacional de clasificación diagnóstico para fines clínicos y de investigación (Organización Mundial de la Salud, s.a.), y que existen otras metodologías para determinar correctamente la incidencia y prevalencia de las enfermedades que no existirían sin la contribución del alcohol (Rehm et al., 2004), las iniciativas exitosas de prevención no están supeditadas a especificar «alcohol», «alcohólico»o «inducido por el alcohol» en el nombre de la enfermedad en la CIE. Consideremos el tabaco como ejemplo: la prevención de la carga de enfermedades atribuibles al tabaquismo sin duda fue posible sin crear categorías diagnósticas como «cáncer de pulmón inducido por el tabaco».

Además, uno de los objetivos principales de clasificar a los pacientes con una u otra enfermedad es vincularlos a la mejor intervención terapéutica posible (Lecrubier, 2008). Si el enfoque del tratamiento no se diferencia de otras condiciones con la misma sintomatología, sea o no idiopática, entonces especificar «alcohol» en el nombre de dicha condición de salud es, sencillamente, innecesario.

Uno podría argumentar que la raíz del problema es la estigmatización y, de hecho, contrario a otros trastornos mentales, la estigmatización del consumo perjudicial de alcohol no ha mejorado en el último par de décadas (Schomerus, Matschinger y Angermeyer, 2014). Por tanto, se requiere urgentemente de esfuerzos para abordar la estigmatización respecto de las condiciones completamente atribuibles al alcohol, y por ello es inaceptable que a día de hoy haya nombres de enfermedades que fomenten la estigmatización. Actualmente tenemos un sistema que lleva a conclusiones imprecisas para los cuidados clínicos, las políticas sobre la salud y la investigación respecto de la mayoría de las condiciones completamente atribuibles al alcohol, cosa que tiene fácil solución. Es hora de que la CIE elimine la especificación etiológica de alcohol en los nombres de las enfermedades que tienen un vínculo causal con el alcohol.

\section{Conflicto de interés}

Los autores declaran la inexistencia de conflicto de interés.

\section{Referencias}

Andresen-Streichert, H., Müller, A., Glahn, A., Skopp, G. y Sterneck, M. (2018). Alcohol biomarkers in clinical and forensic contexts. Deutsches Arzteblatt International, 115, 309-315. doi:10.3238/arztebl.2018.0309.

Carvalho, A. F., Heilig, M., Perez, A., Probst, C. y Rehm, J. (2019). Alcohol use disorders. The Lancet, 394, 781-792. doi:10.1016/S0140-6736(19)31775-1.

Fuster, D. y Samet, J. H. (2018). Alcohol use in patients with chronic liver disease. New England Journal of Medicine, 379, 1251-1261. doi:10.1056/NEJMc1814129.

GBD 2016 Alcohol Collaborators. (2018). Alcohol use and burden for 195 countries and territories, 1990-2016: a systematic analysis for the Global Burden of Disease 
Study 2016. The Lancet, 392, 1015-1035. doi:10.1016/ S0140-6736(18)31310-2.

Lange, S., Rehm, J., Anagnostou, E. y Popova, S. (2018). Prevalence of externalizing disorders and Autism Spectrum Disorders among children with Fetal Alcohol Spectrum Disorder: systematic review and meta-analysis. Biochemistry and Cell Biology, 96, 241-251. doi:10.1139/ bcb-2017-0014.

Lange, S., Shield, K., Koren, G., Rehm, J. y Popova, S. (2014). A comparison of the prevalence of prenatal alcohol exposure obtained via maternal self-reports versus meconium testing: a systematic literature review and meta-analysis. BMC Pregnancy and Childbirth, 14, 127. doi:10.1186/1471-2393-14-127.

Lange, S., Shield, K., Rehm, J., Anagnostou, E. y Popova, S. (2019). Fetal alcohol spectrum disorder: Neurodevelopmentally and behaviorally indistinguishable from other neurodevelopmental disorders. BMC Psychiatry, 19, 322. doi:10.1186/s12888-019-2289-y.

Lecrubier, Y. (2008). Refinement of diagnosis and disease classification in psychiatry. European Archives of Psychiatry and Clinical Neuroscience, 258(Supl. 1), 6-11. doi:10.1007/ s00406-007-1003-0.

Murawski, N. J., Moore, E. M., Thomas, J. D. y Riley, E. P. (2015). Advances in Diagnosis and Treatment of Fetal Alcohol Spectrum Disorders: From Animal Models to Human Studies. Alcohol Research: Current Reviews, 37, 97108.

Organización Mundial de la Salud. (2018a). Classifications ICD-11 2018. Ginebra, Suiza: OMS. Recuperado de https://www.who.int/classifications/icd/en/

Organización Mundial de la Salud. (2018b). Global status report on alcohol and health 2018. Ginebra, Suiza: OMS. Recuperado de https://www.who.int/substance_abuse/ publications/global_alcohol_report/en/

Organización Mundial de la Salud. (s.a.). International Classification of Diseases (ICD) Information Sheet. Recuperado de https://www.who.int/classifications/icd/factsheet/en/

Pimpin, L., Cortez-Pinto, H., Negro, F., Corbould, E., Lazarus, J. V., Webber, L.,... EASL HEPAHEALTH Steering Committee. (2018). Burden of liver disease in Europe: Epidemiology and analysis of risk factors to identify prevention policies. Journal of Hepatology, 69, 718-735. doi:10.1016/j.jhep.2018.05.011.

Premji, S., Benzies, K., Serrett, K. y Hayden, K. A. (2007). Research-based interventions for children and youth with a Fetal Alcohol Spectrum Disorder: revealing the gap. Child: Care, Health and Development, 33, 389-397; discussion 398-400.

Price, K. J. y Miskelly, K. J. (2015). Why Ask Why? Logical Fallacies in the Diagnosis of Fetal Alcohol Spectrum Disorder. Ethics Eै Behavior, 25, 418-426. doi:10.1080/1050 8422.2014.946031
Probst, C., Manthey, J., Martinez, A. y Rehm, J. (2015). Alcohol use disorder severity and reported reasons not to seek treatment: a cross-sectional study in European primary care practices. Substance Abuse Treatment, Prevention, and Policy, 10, 32. doi:10.1186/s13011-015-0028-z.

Puffer, R. R. y Griffith, G. W. (1967). Patterns of urban mortality: report of the Inter-American Investigation of Mortality. Scientific Publication no. 151. Washington, D.C.: Pan American Health Organization.

Rehm, J., Gmel, G. E. Sr., Gmel, G., Hasan, O. S. M., Imtiaz, S., Popova, S.,... Shuper, P. A. (2017). The relationship between different dimensions of alcohol use and the burden of disease-an update. Addiction, 112, 968-1001. doi:10.1111/add.13757.

Rehm, J., Hasan, O. S. M., Imtiaz, S. y Neufeld, M. (2017). Quantifying the contribution of alcohol to cardiomyopathy: a systematic review. Alcohol, 61, 9-15. doi:10.1016/j. alcohol.2017.01.011.

Rehm, J., Room, R., Monteiro, M., Gmel, G., Graham, K., Rehn, N.,... Jernigan, D. (2004). Alcohol use. En: M. Ezzati, A. D. Lopez, A. Rodgers y C. J. L. Murray (Eds.), Comparative quantification of health risks: global and regional burden of disease attributable to selected major risk factors. (pp. 959-1108). Ginebra, Suiza: Organización Mundial de la Salud.

Roerecke, M., Vafaei, A., Hasan, O. S. M., Chrystoja, B., Cruz, M., Lee, R.,... Rehm, J. (2019). Alcohol consumption and risk of liver cirrhosis: a systematic review and meta-analysis. American Journal of Gastroenterology, 114, 1574-1586. doi:10.14309/ajg.0000000000000340.

Schomerus, G., Lucht, M., Holzinger, A., Matschinger, H., Carta, M. G. y Angermeyer, M. C. (2011). The stigma of alcohol dependence compared with other mental disorders: a review of population studies. Alcohol and Alcoholism, 46, 105-112. doi:10.1093/alcalc/agq089.

Schomerus, G., Matschinger, H. y Angermeyer, M. C. (2014). Attitudes towards alcohol dependence and affected individuals: persistence of negative stereotypes and illness beliefs between 1990 and 2011. European Addiction Research, 20, 293-299. doi:10.1159/000362407.

Schwarzinger, M., Baillot, S., Yazdanpanah, Y., Rehm, J. y Mallet, V. (2017). Contribution of alcohol use disorders on the burden of chronic hepatitis C in France, 20082013: A nationwide retrospective cohort study. Journal of Hepatology, 67, 454-461. doi:10.1016/j.jhep.2017.03.031.

Shrivastava, A., Bureau, Y., Rewari, N. y Johnston, M. (2013). Clinical risk of stigma and discrimination of mental illnesses: Need for objective assessment and quantification. Indian Journal of Psychiatry, 55, 178-182. doi:10.4103/0019-5545.111459. 